\title{
Standard completeness for uninorm-based logics
}

\author{
Paolo Baldi \\ Vienna University of Technology, Austria \\ Inst. of Comp. Science, Academy of Sciences, Czech Rep. \\ Email: baldi@logic.at
}

\author{
Agata Ciabattoni \\ Vienna University of Technology, Austria \\ Email: agata@logic.at
}

\begin{abstract}
We provide a uniform proof of standard completeness for a large class of axiomatic extensions of Uninorm Logic.
\end{abstract}

\section{INTRODUCTION}

Logics based on uninorms were introduced in [1] as a generalization of the t-norm based logic approach developed by Hájek [2]. In this approach the real unit interval $[0,1]$ is taken as set of truth values and conjunction "." and implication " $\rightarrow$ " are interpreted as suitable $t$-norms ${ }^{1}$ (or classes of t-norms) and their residua. Introduced in [3] and successfully used in various applications ranging from expert systems to fuzzy modeling, uninorms are a generalization of $t$-norms where the identity element can be any value in $[0,1]$.

Uninorm-based logics arise by extending the logic UL of left-continuous uninorms [1] with suitable Hilbert axioms. This design leads however to a fuzzy logic (in the sense of Hájek) only when the resulting system is standard complete, that is complete w.r.t. algebras whose lattice reduct is $[0,1]$. Proving that is often challenging, especially for axiomatic extensions of UL that do not contain weakening/integrality (i.e. not extending the logic of left continuous t-norms MTL [4]). Standard completeness has been indeed established for a handful such logics, namely UL with the addition of contraction $\alpha \rightarrow \alpha^{2}$ and mingle $\alpha^{2} \rightarrow \alpha$ ( $\alpha^{k}$ stands for $\alpha \cdot \alpha \cdots \alpha k$ times) in the seminal paper [1], UL with n-contraction $\alpha^{n-1} \rightarrow \alpha^{n}$ and n-mingle $\alpha^{n} \rightarrow \alpha^{n-1}(n>2)$ in [5], and UL with knotted axioms $\alpha^{k} \rightarrow \alpha^{j}$ (for $j, k>1$ ) in [6]. These proofs are all tailored to the specific axiomatic extensions considered and seem hard to generalize. The main idea behind the proofs in [1], [6] - which use proof theoretic methods - is that the addition of a special rule, called density, to any axiomatic extension of UL makes the resulting logic rational complete, i.e. complete with respect to algebras over the rationals in $[0,1]$. Thus, the admissibility (or elimination) of the density rule entails rational completeness for the original logic. Once this has been established, standard completeness follows (in many cases) by means of the Dedekind-MacNeille completion. In this paper we present a proof of standard completeness that uniformly applies to many extensions of UL with axioms in the class $\mathcal{N}_{2}$ of the syntactic classification of [7]. Its core is a general proof of the elimination of the density rule in hypersequent calculi extending that for UL [1] with a host of

\footnotetext{
${ }^{1} \mathrm{~T}$-norms are commutative, associative, monotonic binary functions with identity 1 .
}

(rules capturing a large class of $\mathcal{N}_{2}$ ) axioms. Our proof applies to all logics lying between UL and MTL already known to be standard complete and allows for the discovery of new uninorm-based fuzzy logics. The latter include UL extended with contraction or mingle, and UL with $f$-knotted axioms $f \cdot \alpha^{k} \rightarrow \alpha^{n}(k \geq 1, n>1)$.

\section{PROOF THEORY FOR AXIOMATIC EXTENSIONS OF UL}

Uninorm logic UL [1] is based on a propositional language with propositional variables, the constants $T, \perp, t, f$ and the connectives $\cdot, \wedge, \vee$ and $\rightarrow$. A Hilbert-system for UL is obtained by adding the prelinearity axiom $((\alpha \rightarrow \beta) \wedge t) \vee((\beta \rightarrow \alpha) \wedge t)$ to any Hilbert calculus for MAILL (the multiplicative-additive fragment of intuitionistic linear logic), see e.g. [8], [9]. A Gentzen-style calculus for UL was introduced in [1] and is based on (single-conclusioned) hypersequents.

Definition 1: [10] A (single-conclusioned) hypersequent is a finite multiset $\Gamma_{1} \Rightarrow \Psi_{1}|\ldots| \Gamma_{n} \Rightarrow \Psi_{n}$ of sequents, where for each $i=1, \ldots, n, \Gamma_{i}$ is a multiset of formulas and $\Psi_{i}$ is either empty or a single formula. $\Gamma_{i} \Rightarrow \Psi_{i}$ is called a component of the hypersequent.

Notation. In the following, hypersequents will be denoted by $G, H$ and sequents (possibly built from metavariables) by $S_{i}, C_{i}$. Within a sequent $S:=\Gamma \Rightarrow \Psi$ we will denote by $L(S)$ the multiset $\Gamma$ in its left hand side, and by $R(S)$ its right hand side $\Psi$. The notation $\Gamma^{k}$ stands for $k$ commaseparated occurrences $\Gamma, \ldots, \Gamma$ of a multiset $\Gamma . \odot \Gamma$ stands for the multiplicative conjunction - of all the formulas in $\Gamma$, and it is $t$ when $\Gamma$ is empty.

The hypersequent calculus HUL for UL is displayed in Table I. Notice that its rules are actually rule schemes and that the (cut) rule is redundant (eliminable, in fact). The rule (com), which operates on different components of hypersequents, allows us to prove the prelinearity axiom (which cannot be captured by a sequent structural rule [7]). A concrete instance of a rule scheme will be referred to as a rule application. Following standard practice, we do not always distinguish explicitly between a rule instance and a rule schema. Given a rule $(r)$, we call active the components of the premises and of the conclusion on which $(r)$ operates. We denote multiple applications of a rule $(r)$ by $(\bar{r})$.

Defining hypersequent calculi admitting cut-elimination for axiomatic extensions of a logic is in general a difficult task. This task was automated in [11] for extensions of UL with large classes of axioms of a certain shape. We recall below 


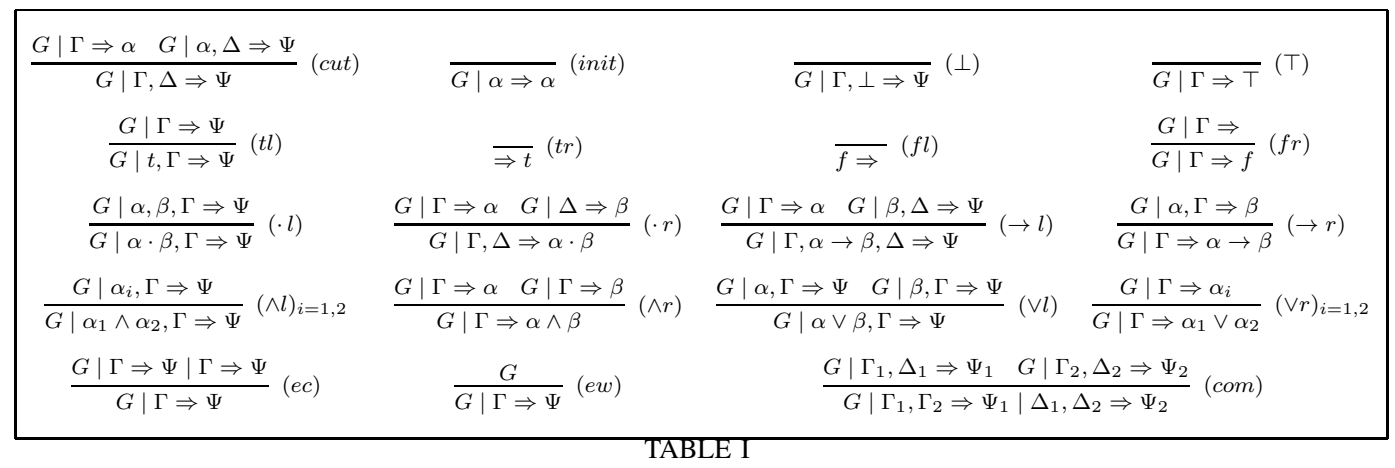

HYPERSEQUENT CALCULUS HUL FOR UL

the class of acyclic $\mathcal{N}_{2}$ axioms from [11], define the proper subclass of nonlinear axioms and the corresponding rules obtained by using the algorithm in [7].

Definition 2: [11] A UL formula $\alpha$ is in the class $\mathcal{N}_{2}$ if $\alpha=\bigwedge_{1 \leq i \leq n} \delta_{i}$, and every $\delta_{i}$ is $\alpha_{1} \cdots \alpha_{m} \rightarrow \beta$ where

- $\beta=f$ or $\beta_{1} \vee \cdots \vee \beta_{k}$ and each $\beta_{l}$ is a multiplicative conjunction of propositional variables or $t$.

- $\alpha_{i}=\bigwedge_{1 \leq j \leq p} \gamma_{i}^{j} \rightarrow \beta_{i}^{j}$ where $\beta_{i}^{j}=f$ or a propositional variable, and $\gamma_{i}^{j}$ is a multiplicative conjunction or a disjunction of propositional variables (or $t$ ).

A formula in $\mathcal{N}_{2}$ is acyclic if for each $\delta_{i}$ the following condition holds: for no propositional variable $p$ (and multiset of formulas $\Pi$ ) the sequent $\Pi, p \Rightarrow p$ arise applying (cut) to $\left\{\gamma_{i}^{j} \vdash \beta_{i}^{j}\right\}_{j=1, \ldots, p}$ in all possible ways.

A nonlinear formula is an acyclic $\mathcal{N}_{2}$ formula in which no propositional variable appears only once in any of the $\beta_{l}, \gamma_{i}^{j}$.

Lemma 1: Let $\mathcal{A}$ be a set of acyclic $\mathcal{N}_{2}$ axioms. (1) There is a set $R$ of hypersequent structural rules such that a formula $\gamma$ is derivable in the Hilbert system for UL $+\mathcal{A}$ iff $\Rightarrow \gamma$ is cut-free derivable in the hypersequent calculus HUL extended with $R$. Moreover, (2) each rule $(r) \in R$ has the form:

$$
\frac{G\left|S_{1} \quad \ldots G\right| S_{m}}{G \mid \Pi, \Gamma_{1}, \ldots, \Gamma_{n} \Rightarrow \Psi}(r)
$$

and satisfies the following properties:

(a) Each metavariable occurring in $L\left(S_{i}\right)$ (respectively in $R\left(S_{i}\right)$ ), occurs in the left (respectively right) hand side of the conclusion

(b) All metavariables $\Pi, \Gamma_{1}, \ldots \Gamma_{n}, \Psi$ are distinct

(c) Whenever $R\left(S_{i}\right)=\Psi$, the metavariable $\Pi \in L\left(S_{i}\right)$.

For any nonlinear axiom in $\mathcal{A}$, the corresponding rule $(r) \in R$ satisfies in addition

(d) For any premise $G \mid S_{i}$ of $(r)$ s.t. $R\left(S_{i}\right) \neq \emptyset$, none of the multisets $\Gamma_{1}, \ldots, \Gamma_{n}$ appears only once in $L\left(S_{i}\right)$

Proof: Claim (1) and the fact that $(r)$ satisfies (a)-(c) follow by the results in [7]. To show that, for nonlinear axioms, the rules obtained by using the algorithm in [7] also satisfy (d), we sketch below the algorithm. This is based on applying backwards the invertible rules of HUL (i.e. $(t l),(f r),(\cdot l)$, $(\wedge, r),(\vee l)$ and $(\rightarrow r))$ as much as possible and on the following observation (known as Ackermann's lemma): The rule $\frac{S_{1} \ldots S_{m}}{\psi_{1}, \ldots, \psi_{n} \Rightarrow \xi}\left(r^{\prime}\right)$ is interderivable -using (init) and (cut) - with each of the rules

$$
\frac{\vec{S} \Lambda_{1} \Rightarrow \psi_{1} \cdots \Lambda_{n} \Rightarrow \psi_{n}}{\Lambda_{1}, \ldots, \Lambda_{n} \Rightarrow \xi}\left(r_{1}\right) \frac{\vec{S} \quad \Pi, \xi \Rightarrow \Psi}{\psi_{1}, \ldots, \psi_{n}, \Pi \Rightarrow \Psi}\left(r_{2}\right)
$$

where $\vec{S}=S_{1} \ldots S_{m}$ and $\Lambda_{1}, \ldots, \Lambda_{n}, \Pi$ are fresh metavariables for multisets of formulas and $\Psi$ is either a formula or the empty set. Let $\alpha=\bigwedge_{1 \leq i \leq n} \delta_{i}$ be a nonlinear axiom. We start with $\Rightarrow \alpha$ and, applying backwards $(\wedge r)$ we get $n$ sequents $\Rightarrow \delta_{i}$ that, as shown below, all give rise to structural rules satisfying (d). Let $\delta_{i}$ be $\alpha_{1} \cdots \alpha_{m} \rightarrow \beta$ as in Definition 2. By applying backwards $(\rightarrow r)$ we get $\alpha_{1}, \ldots, \alpha_{m} \Rightarrow \beta$. Assume that $\beta \neq f$ (as otherwise we can simply remove $f$ by $(f r)$ ), Ackermann's lemma and subsequent applications of the invertible rules give

$$
\frac{\left\{\Pi, \beta_{j} \Rightarrow \Psi\right\}_{j=1, \ldots, k} \quad\left\{\Gamma_{i}, \gamma_{i}^{j} \Rightarrow \beta_{i}^{j}\right\}_{j=1, \ldots, p}}{\Gamma_{1} \cdots \Gamma_{m}, \Pi \Rightarrow \Psi}
$$

By applying backwards the invertible rules for $t$ and $f$ together with $(\cdot l)$ to all $\beta_{j}$ and $(\vee l)$ and $(\cdot l)$ to all $\gamma_{i}^{j}$ we remove all connectives and constants from the premises. Now if there are propositional variables that appear in the premises only on the same side, then the premises containing that variables are simply removed. We cut the remaining premises in all possible ways. Since the propositional variables on the left hand side of the premises appear all with multiplicities ( $\alpha$ being a nonlinear axiom), the resulting rule satisfies (d).

Henceforth we call nonlinear, the hypersequent rules satisfying conditions (a)-(d) in the above lemma.

Example 1: The rules $(c),\left(k_{n o t}^{n}\right),\left(f k n o t_{k}^{n}\right)$ for $n>1$ (and corresponding axioms) in Table II are nonlinear. This is not the case of mingle $(m g l)$ and $\left(k n o t_{k}^{1}\right)$.

\section{DENSITY ELIMINATION}

We now consider the extension of our hypersequent calculi with the density rule. This rule, first introduced Hilbert-style in [12], will be considered here as

$$
\frac{G|\Lambda \Rightarrow p| p \Rightarrow \Delta}{G \mid \Lambda \Rightarrow \Delta}(D)
$$

where $p$ is an eigenvariable, i.e. $p$ should not appear in the lower hypersequent. Ignoring $G,(D)$ can be read contrapositively as saying (very roughly) "if $\Lambda>\Delta$, then $\Lambda>p$ and $p>\Delta$ for some $p$ "; hence the name "density". 


\begin{tabular}{|l|l|}
\hline Axiom & Rule generated by the algorithm in [7] \\
\hline \multirow{2}{*}{$\alpha \rightarrow \alpha^{2}$} & $\frac{G_{1} \mid \Pi, \Gamma_{1}, \Gamma_{1} \Rightarrow \Psi}{G_{1} \mid \Pi, \Gamma_{1} \Rightarrow \Psi}(c)$ \\
$\alpha^{2} \rightarrow \alpha$ & $\frac{G_{1}\left|\Pi, \Gamma_{1} \Rightarrow \Psi \quad G_{1}\right| \Pi, \Gamma_{2} \Rightarrow \Psi}{G_{1} \mid \Pi, \Gamma_{1}, \Gamma_{2}, \Rightarrow \Psi}(m g l)$ \\
$\alpha^{k} \rightarrow \alpha^{n}$ & $\frac{G_{1}\left|\Pi, \Gamma_{1}^{n} \Rightarrow \Psi \ldots G_{1}\right| \Pi, \Gamma_{k}^{n} \Rightarrow \Psi}{G_{1} \mid \Pi, \Gamma_{1}, \ldots \Gamma_{k} \Rightarrow \Psi}\left(k n o t_{k}^{n}\right)$ \\
$f \cdot \alpha^{k} \rightarrow \alpha^{n}$ & $\frac{\left\{G_{1} \mid \Pi, \Gamma_{i}^{n} \Rightarrow \Psi\right\}_{i=1, \ldots k} \quad G_{1} \mid \Gamma_{k+1} \Rightarrow}{G_{1} \mid \Pi, \Gamma_{1}, \ldots \Gamma_{k}, \Gamma_{k+1} \Rightarrow \Psi}\left(\right.$ fknot $\left._{k}^{n}\right)$ \\
\hline
\end{tabular}

SOME $\mathcal{N}_{2}$ AXIOMS AND THE CORRESPONDING STRUCTURAL RULES

In this section we prove density-elimination for any hypersequent calculus extending HUL with nonlinear rules and possibly $(\mathrm{mgl})$ (cf. Table II). This means that any derivation containing an application of $(D)$ can be transformed into a derivation of the same end-hypersequent which does not contain $(D)$. Our proof generalizes that in [11] which is applied to a small subset of the calculi/logics we can deal with. To this end, we first recall the idea behind the density elimination proof in [11]. Assume we have a derivation $d$ ending in an application of $(D)$ as the one above. By Lemma 1 we can safely assume $d$ to be cut-free. We remove the application of $(D)$ by substituting all occurrences of $p$ in $d$ in an "asymmetric" way. More precisely, each $p$ occurring on the left hand side of a sequent is replaced by $\Lambda$, and each $p$ on the right hand side by $\Delta$. The above application of $(D)$ would then be simply replaced by $(e c)$. However, this procedure in general does not result in a "correct" derivation anymore. For instance if $d$ contains an axiom $p \Rightarrow p$, the asymmetric substitution would transform it into $\Lambda \Rightarrow \Delta$, that is not an axiom. The idea is then to replace each component of the form $\Pi, p^{k} \Rightarrow p$ by $\Pi, \Lambda^{k-1} \Rightarrow t$ (and hence $p \Rightarrow p$ is simply $\Rightarrow t$ ), perform the asymmetric substitution in all other hypersequents in the derivation and add suitable cuts to handle problematic applications of $(\mathrm{com})$. This method, originally introduced in [11] to deal with extensions of HUL with sequent structural rules having the same number of occurrences of metavariables in the premises and in the conclusion, was applied in [6] to deal with HUL with the rules $\left(k n o t_{k}^{n}\right)$ for $n, k>1$. The method however does not work, e.g. for important structural rules such as $(c)$. Consider indeed the case in which the derivation $d$ (below left) contains an application of $(c)$. After the substitution we get the derivation below right, where the "incorrect" application of $(c)$ is marked with (?).

$$
\begin{array}{cc}
p \Rightarrow p & \Rightarrow t \\
\vdots & \vdots \\
\frac{\Pi, p, p \Rightarrow p}{\Pi, p \Rightarrow p}(c) & \frac{\Pi, \Lambda \Rightarrow t}{\Pi \Rightarrow t}(?) \\
\frac{G|\Lambda \Rightarrow p| p \Rightarrow \Delta}{G \mid \Lambda \Rightarrow \Delta}(D) & \frac{G|\Lambda \Rightarrow \Delta| \Lambda \Rightarrow \Delta}{G \mid \Lambda \Rightarrow \Delta}(e c)
\end{array}
$$

To solve the problem we look back at the whole original derivation $d$, with the additional knowledge that $\Pi, \Lambda \Rightarrow t$ is derivable (this is always the case if we start the proof transformation from the uppermost application of $(c)$ in $d$ ). The idea is to perform a new substitution: recall that an axiom $p \Rightarrow p$ should be replaced with something derivable. This time, instead of $\Rightarrow t$, we let the sequent $\Pi, \Lambda \Rightarrow t$ do the job. More precisely, we perform the following substitution to the whole derivation $d$ : we replace each $\Sigma, p^{k} \Rightarrow p$ with $\Sigma, \Lambda^{k-1}, \Pi, \Lambda \Rightarrow t$ and in all other sequents any $p$ occurring on the left with $\Lambda$ and any $p$ occurring on the right with $\Delta$. As shown below this new substitution eventually leads to a derivation $d_{1}$ of $G|\Lambda \Rightarrow \Delta| \Pi, \Lambda, \Lambda \Rightarrow \Delta$. The (?) in the derivation above will thus be replaced by the subderivation

$$
\begin{gathered}
\Pi, \Lambda \Rightarrow t \\
\vdots d_{1} \\
\frac{{ }^{G|\Lambda \Rightarrow \Delta| \Pi, \Lambda, \Lambda \Rightarrow \Delta}}{G|\Lambda \Rightarrow \Delta| \Pi, \Lambda \Rightarrow \Delta \quad(c) \quad \Rightarrow t}(\mathrm{com})
\end{gathered}
$$

Notice that the additional components $G \mid \Lambda \Rightarrow \Delta$ can be removed by applications of $(e c)$ at the end of our restructured derivation. This procedure is formalized in the following. We start by recalling a technical lemma needed for our density elimination proof.

Lemma 2: [13] Let HL be a hypersequent calculus extending HUL with any rule obtained by the algorithm in [7]. Let $d$ be a cut-free, density-free derivation of $G|p \Rightarrow \Delta| \Lambda \Rightarrow p(p \notin$ $G, \Delta, \Lambda)$. The following rule is derivable in $\mathrm{HL}$

$$
\frac{G \mid \Theta, \Pi \Rightarrow \Psi}{G|\Theta, \Lambda \Rightarrow \Psi| \Pi \Rightarrow \Delta}\left(\text { split }_{d}\right)
$$

Notation. Let $S$ be a sequent. We denote with

$$
S\left[{ }^{p \Rightarrow p} / \Lambda \Rightarrow \Delta\right]
$$

the sequent obtained by replacing one occurrence of $p$ on $L(S)$ with $\Lambda$ and $R(S)$ with $\Delta$, if $R(S)=p$. We use the notation

$$
S\left[^{\bar{p} \Rightarrow p} / \Lambda \Rightarrow \Delta\right]
$$

for the substitution of each occurrence of $p$ in $L(S)$ with a $\Lambda$. As an example, for $S:=\Pi, p^{k} \Rightarrow p$, we have $S\left[{ }^{p \Rightarrow p} / \Lambda \Rightarrow \Delta\right]=$ $\Pi, \Lambda, p^{k-1} \Rightarrow \Delta$, and $S\left[{ }^{\bar{p} \Rightarrow p} / \Lambda \Rightarrow \Delta\right]=\Pi, \Lambda^{k} \Rightarrow \Delta$.

Sequents of the form $\Pi, p^{k} \Rightarrow p$ will be called $p p$ components in the following.

Theorem 1: Let HL be any hypersequent calculus extending HUL with any nonlinear rule and possibly $(m g l)$. The calculus $\mathrm{HL}+(D)$ admits density elimination.

Proof: Let $d$ be a $(c u t),(D)$-free derivation ending in

$$
\frac{G|\Lambda \Rightarrow p| p \Rightarrow \Delta}{G \mid \Lambda \Rightarrow \Delta}(D)
$$

We show that we can get a $(D)$-free derivation of $G \mid \Lambda \Rightarrow \Delta$. Let $H$ be $H=S_{1}|\ldots| S_{n}$, we define $H^{*}$ as $S_{1}^{*}|\ldots| S_{n}^{*}$ where for each $i=1, \ldots, n$

- $S_{i}^{*}=\left(S_{i}[\stackrel{p \Rightarrow p}{[} / \Rightarrow t]\right)\left[{ }^{\bar{p} \Rightarrow} / \Lambda \Rightarrow\right]$, if $S_{i}$ is a $p p$-component. 
- $S_{i}^{*}=S_{i}\left[{ }^{\bar{p} \Rightarrow p} / \Lambda \Rightarrow \Delta\right]$ otherwise

We prove the following by induction

\section{Claim : For each hypersequent $H$ in $d$ we can find a (D)-free derivation of $G|\Lambda \Rightarrow \Delta| H^{*}$}

Density elimination follows by applying the claim to the hypersequent $G|\Lambda \Rightarrow p| p \Rightarrow \Delta$. We have indeed $G \mid \Lambda \Rightarrow$ $\Delta\left|(G|\Lambda \Rightarrow p| p \Rightarrow \Delta)^{*}=G\right| \Lambda \Rightarrow \Delta|G| \Lambda \Rightarrow \Delta \mid \Lambda \Rightarrow \Delta$. (Observe that $G^{*}=G$ by the eigenvariable condition on $p$ ). The desired hypersequent is obtained by $(\overline{e c})$. All the cases, but the ones involving nonlinear rules or $(m g l)$, are treated as in [11]. Let $(r)$ be the last rule applied in $d$ to derive $H$. We distinguish cases according to the active $p p$-components of the rule application. In case the conclusion of $(r)$ is not a $p p$-component, by the property (a) of Lemma 1 , none of the premises is a $p p$-component. The claim simply follows by the i.h. and an application of $(r)$. Assume that $(r)$ is a non linear rule and its conclusion is a $p p$-component as in

$$
\frac{G_{1}\left|S_{1} \ldots G_{1}\right| S_{m}}{G_{1} \mid \Pi, \Gamma_{1}, \ldots, \Gamma_{n}, p^{k} \Rightarrow p}(r)
$$

If none of the premises is a $p p$-component, we can simply apply the i.h. and consider the following

$$
\frac{G|\Lambda \Rightarrow \Delta| G_{1}^{*}\left|S_{1}^{*} \quad \ldots \quad G\right| \Lambda \Rightarrow \Delta\left|G_{1}^{*}\right| S_{m}^{*}}{\frac{G|\Lambda \Rightarrow \Delta| G_{1}^{*} \mid \Pi, \Gamma_{1}, \ldots, \Gamma_{n}, \Lambda^{k} \Rightarrow \Delta}{(r) \quad \Rightarrow t}}(\mathrm{com})
$$

Assume now that the conclusion of $(r)$ is a $p p$-component and the metavariable $\Pi$, which witnesses for $(r)$ the property (c) in Lemma 1 is instantiated with at least one $p$. By the properties (a) and (c), all the premises of $(r)$ are either of the form $\Theta_{i}, p^{n_{i}} \Rightarrow p$, for $n_{i} \geq 1$ or $\Theta_{i} \Rightarrow$. Consequently any $S_{i}^{*}$ will be either of the form $\Theta_{i}, \Lambda^{n_{i}-1} \Rightarrow t$ or $\Theta_{i} \Rightarrow$. The claim just follows by applying the rule $(r)$ to all the $S_{i}^{*}$ 's.

Assume now that $\Pi$ is not instantiated with any $p$ and that at least some $S_{i}$, say those for $i \in\{1, \ldots, q\}$, are $p p$-components. Thus, by property (d), $p p$-components should necessarily contain more than one $p$, i.e. we can assume that any $S_{i}$, for $i \in\{1, \ldots, q\}$ is of the form $\Pi, \Theta_{i}, p^{n_{i}} \Rightarrow p$, with $n_{i} \geq 2$. In addition, we might have premises of the form $\Pi, \Theta_{i} \Rightarrow p$ or $\Theta_{i} \Rightarrow$. The sequents $S_{i}^{*}$ will be of the form $\Pi, \Theta_{i}, \Lambda^{n_{i}-1} \Rightarrow t$ or $\Pi, \Theta_{i} \Rightarrow \Delta$ or $\Theta_{i} \Rightarrow$, respectively. By the i.h., we have derivations of $G|\Lambda \Rightarrow \Delta| G_{1}^{*}\left|S_{1}^{*}, \ldots, G\right| \Lambda \Rightarrow \Delta\left|G_{1}^{*}\right| S_{m}^{*}$, which cannot be used as premises of $(r)$. We instead apply Lemma 3 below to each of the $G|\Lambda \Rightarrow \Delta| G_{1}^{*} \mid S_{i}^{*}$, for $i=1, \ldots q$, thus obtaining $G|\Lambda \Rightarrow \Delta| G_{1}^{*} \mid \Pi, \Theta_{i}, \Lambda^{n_{i}} \Rightarrow \Delta$. We then apply the rule $(r)$ as follows.

$$
\begin{aligned}
& \left\{G|\Lambda \Rightarrow \Delta| G_{1}^{*} \mid \Pi, \Theta_{i}, \Lambda^{n_{i}} \Rightarrow \Delta\right\}_{i=1, \ldots, q} \\
& \frac{\left\{G|\Lambda \Rightarrow \Delta| G_{1}^{*} \mid S_{i}^{*}\right\}_{i=q+1, \ldots, m}}{\frac{G|\Lambda \Rightarrow \Delta| G_{1}^{*} \mid \Pi, \Gamma_{1}, \ldots, \Gamma_{n}, \Lambda^{k} \Rightarrow \Delta}{(r) \Rightarrow t}}\left(\begin{array}{l}
G|\Lambda \Rightarrow \Delta| G_{1}^{*}\left|\Pi, \Gamma_{1}, \ldots, \Gamma_{n}, \Lambda^{k-1} \Rightarrow t\right| \Lambda \Rightarrow \Delta \\
G|\Lambda \Rightarrow \Delta| G_{1}^{*} \mid \Pi, \Gamma_{1}, \ldots, \Gamma_{n}, \Lambda^{k-1} \Rightarrow t
\end{array}(\mathrm{ec})\right.
\end{aligned}
$$

The application of $(r)$ above is correct, being nothing more (apart from the hypersequent context) than the original rule application in which every premise $S_{i}$ is replaced by $S_{i}\left[{ }^{\bar{p} \Rightarrow p} / \Lambda_{\Rightarrow \Delta}\right]$. Assume that the last rule applied is $(m g l)$ and that its conclusion contains a $p p$-component. Two subcases can appear: both premises of $(m g l)$ are $p p$-component or only one of the premises is. The latter case can be reduced to the former. Indeed, assume that we have

$$
\frac{G_{1}\left|\Pi, \Gamma_{1} \Rightarrow p \quad G_{1}\right| \Pi, \Gamma_{2}, p \Rightarrow p}{G_{1} \mid \Pi, \Gamma_{1}, \Gamma_{2}, p \Rightarrow p}(m g l)
$$

We apply $(m g l)$ with premises $G_{1} \mid \Pi, \Gamma_{2}, p \Rightarrow p$, thus getting $G_{1} \mid \Pi, \Gamma_{2}, \Gamma_{2}, p, p \Rightarrow p$. Similarly, we obtain $G_{1} \mid \Pi, \Gamma_{1}, \Gamma_{1} \Rightarrow p$ from $G_{1} \mid \Pi, \Gamma_{1} \Rightarrow p$. Hence we get

$$
\frac{G_{1}\left|\Pi, \Gamma_{1}, \Gamma_{1} \Rightarrow p \quad G_{1}\right| \Pi, \Gamma_{2}, \Gamma_{2}, p, p \Rightarrow p}{\frac{G_{1}\left|\Pi, \Gamma_{1}, \Gamma_{2}, p \Rightarrow p\right| \Pi, \Gamma_{1}, \Gamma_{2}, p \Rightarrow p}{G_{1} \mid \Pi, \Gamma_{1}, \Gamma_{2}, p \Rightarrow p}(\mathrm{ec})}(\mathrm{com})
$$

Assume that both premises contain $p p$-components, e.g., as in

$$
\frac{G_{1}\left|\Pi, \Gamma_{1}, p \Rightarrow p \quad G_{1}\right| \Pi, \Gamma_{2}, p \Rightarrow p}{G_{1} \mid \Pi, \Gamma_{1}, \Gamma_{2}, p, p \Rightarrow p}(m g l)
$$

W.l.o.g. we assume $G_{1}=G_{1}^{*}=\emptyset$. By i.h. we have derivations of $G|\Lambda \Rightarrow \Delta| \Pi, \Gamma_{1} \Rightarrow t$ and $G|\Lambda \Rightarrow \Delta| \Pi, \Gamma_{2} \Rightarrow t$. Consider the following derivation $d_{1}$

$$
\begin{aligned}
& \frac{G|\Lambda \Rightarrow \Delta| \Pi, \Gamma_{1} \Rightarrow t \quad G|\Lambda \Rightarrow \Delta| \Pi, \Gamma_{1} \Rightarrow t}{G|\Lambda \Rightarrow \Delta| \Pi^{2}, \Gamma_{1}^{2} \Rightarrow t}(m g l) \\
& \frac{\frac{G|\Lambda \Rightarrow \Delta| \Pi, \Gamma_{1}, \Lambda \Rightarrow t \mid \Pi, \Gamma_{1} \Rightarrow \Delta}{\left.G \mid \Lambda \Rightarrow \Delta p l i t_{d}\right)}}{\frac{G\left(\Pi, \Gamma_{1}, \Lambda \Rightarrow t \mid \Pi, \Gamma_{1} \Lambda \Rightarrow \Delta\right.}{G|\Lambda \Rightarrow \Delta| \Pi, \Gamma_{1}, \Gamma_{2}, \Lambda \Rightarrow t \mid \Pi, \Gamma_{1}, \Lambda \Rightarrow \Delta} \quad G|\Lambda \Rightarrow \Delta| \Pi, \Gamma_{2} \Rightarrow t}
\end{aligned}
$$

Where $(*)$ stands for an application of Lemma 4 below. Similarly, we can obtain a derivation $d_{2}$ of $G|\Lambda \Rightarrow \Delta| \Pi, \Gamma_{1}, \Gamma_{2}, \Lambda \Rightarrow$ $t \mid \Pi, \Gamma_{2}, \Lambda \Rightarrow \Delta$. Applying $(m g l)$ to the end-hypersequent of $d_{1}$ and $d_{2}$ we obtain a derivation of $G|\Lambda \Rightarrow \Delta| \Pi, \Gamma_{1}, \Gamma_{2}, \Lambda \Rightarrow$ $t \mid \Pi, \Gamma_{1}, \Gamma_{2}, \Lambda^{2} \Rightarrow \Delta$. The desired hypersequent is finally obtained as follows

$$
\frac{\frac{G|\Lambda \Rightarrow \Delta| \Pi, \Gamma_{1}, \Gamma_{2}, \Lambda \Rightarrow t \mid \Pi, \Gamma_{1}, \Gamma_{2}, \Lambda^{2} \Rightarrow \Delta \Rightarrow t}{G|\Lambda \Rightarrow \Delta| \Pi, \Gamma_{1}, \Gamma_{2}, \Lambda \Rightarrow t\left|\Pi, \Gamma_{1}, \Gamma_{2}, \Lambda \Rightarrow t\right| \Lambda \Rightarrow \Delta}}{G|\Lambda \Rightarrow \Delta| \Pi, \Gamma_{1}, \Gamma_{2}, \Lambda \Rightarrow t}(\overline{c o m})
$$

Lemma 3: Let HL and $d$ be as in Theorem 1 and assume to have a derivation of a hypersequent $G_{1} \mid \Pi, \Lambda^{k-1} \Rightarrow t$, where $k \geq 2$, and no $p$ appears. We can find a $(D)$-free derivation of the hypersequent $H_{1}=G|\Lambda \Rightarrow \Delta| G_{1} \mid \Pi, \Lambda^{k} \Rightarrow \Delta$

Proof:

Let $H=S_{1}|\ldots| S_{n}$. We define $H^{* *}$ as $S_{1}^{* *}|\ldots| S_{n}^{* *}$ where for each $i=1, \ldots, n$

- $\left.S_{i}^{* *}=\left(S_{i}\left[{ }^{p \Rightarrow p} / \Pi, \Lambda^{k-1} \Rightarrow t\right]\right){ }^{p} \Rightarrow / \Lambda \Rightarrow\right]$ if $S_{i}$ is a $p p$ component.

- $\left.S_{i}^{* *}=S_{i}{ }^{[\bar{p} \Rightarrow p} / \Lambda \Rightarrow \Delta\right]$ otherwise

Our statement is a consequence of the following 
Claim: For each hypersequent $H$ in $d$ we can find $a(D)$-free derivation of $H_{1} \mid H^{* *}$

We prove the claim by induction on the length of the derivation $d$. Let $H$ be the axiom $p \Rightarrow p$. Recall that the hypersequent $G_{1} \mid \Pi, \Lambda^{k-1} \Rightarrow t$ is derivable by assumption. Hence, applying $(\overline{e w})$ to the latter, we obtain $H_{1}\left|(p \Rightarrow p)^{* *}=G\right| \Lambda \Rightarrow$ $\Delta\left|G_{1}\right| \Pi, \Lambda^{k} \Rightarrow \Delta \mid \Pi, \Lambda^{k-1} \Rightarrow t$. Logical rules (but $(\rightarrow l)$ ), $(e c)$ and $(e w)$ are easy to handle. The case of $(\rightarrow l)$ with active $p p$-component in the conclusion and no $p p$-component in the active premises is handled by using $(\mathrm{com})$.

Assume now that the last rule applied in a derivation of $H$ is $(\mathrm{com})$. In case neither the premises nor the conclusion contain active $p p$-components, the claim is straightforward. We discuss below all non-trivial cases. First, we assume that the conclusion of $(\mathrm{com})$ does not contain any active $p p$ component, while one of the premises does, as in:

$$
\frac{G_{2}\left|\Gamma_{1}, \Gamma_{2}, p \Rightarrow p \quad G_{2}\right| \Pi_{1}, \Pi_{2} \Rightarrow \Delta_{1}}{G_{2}\left|\Gamma_{1}, \Pi_{1}, p \Rightarrow \Delta_{1}\right| \Gamma_{2}, \Pi_{2} \Rightarrow p} \text { (com) }
$$

Our aim is to get a $(D)$-free derivation of $H_{1}\left|G_{2}^{* *}\right| \Gamma_{1}, \Pi_{1}, \Lambda \Rightarrow$ $\Delta_{1} \mid \Gamma_{2}, \Pi_{2} \Rightarrow \Delta$. By i.h. we have derivations of $H_{1}\left|G_{2}^{* *}\right| \Pi_{1}, \Pi_{2} \Rightarrow \Delta_{1}$ and $H_{1}\left|G_{2}^{* *}\right| \Gamma_{1}, \Gamma_{2}, \Pi, \Lambda^{k-1} \Rightarrow t$. The desired hypersequent is obtained as follows (the application of $(e c)$ is justified as $\Pi, \Lambda^{k} \Rightarrow \Delta$ is a component of $H_{1}$ )

$$
\frac{H_{1}\left|G_{2}^{* *}\right| \Gamma_{1}, \Gamma_{2}, \Pi, \Lambda^{k-1} \Rightarrow t \quad \frac{H_{1}\left|G_{2}^{* *}\right| \Pi_{1}, \Pi_{2} \Rightarrow \Delta_{1}}{H_{1}\left|G_{2}^{* *}\right| \Pi_{1}, \Pi_{2}, t \Rightarrow \Delta_{1}}(t l)}{\frac{H_{1}\left|G_{2}^{* *}\right| \Gamma_{1}, \Gamma_{2}, \Pi_{1}, \Pi_{2}, \Pi, \Lambda^{k-1} \Rightarrow \Delta_{1}}{H_{1}\left|G_{2}^{* *}\right| \Gamma_{1}, \Pi_{1}, \Pi, \Lambda^{k} \Rightarrow \Delta_{1} \mid \Gamma_{2}, \Pi_{2} \Rightarrow \Delta}\left(\text { split }_{d}\right)}(\text { cut })
$$

The case of an application of (com) with two $p p$ components in the premises and one in the conclusion is similar. Assume now that the conclusion of (com) contains one active $p p$-component, while none of the premises does

$$
\frac{G_{2}\left|\Gamma_{1}, \Gamma_{2}, p \Rightarrow \Delta_{1} \quad G_{2}\right| \Pi_{1}, \Pi_{2} \Rightarrow p}{G_{2}\left|\Gamma_{1}, \Pi_{1} \Rightarrow \Delta_{1}\right| \Gamma_{2}, \Pi_{2}, p \Rightarrow p}(\mathrm{com})
$$

Our aim is to get a $(D)$-free derivation of $H_{1}\left|G_{2}^{* *}\right| \Gamma_{1}, \Pi_{1} \Rightarrow$ $\Delta_{1} \mid \Gamma_{2}, \Pi_{2}, \Pi, \Lambda^{k-1} \Rightarrow t$. By i.h. we have derivations of $H_{1}\left|G_{2}^{* *}\right| \Gamma_{1}, \Gamma_{2}, \Lambda \Rightarrow \Delta_{1}$ and $H_{1}\left|G_{2}^{* *}\right| \Pi_{1}, \Pi_{2} \Rightarrow \Delta$. We obtain the desired hypersequent as follows (the application of $(e c)$ is justified as $\Lambda \Rightarrow \Delta$ is a component of $H_{1}$ )

$$
\frac{H_{1}\left|G_{2}^{* *}\right| \Gamma_{1}, \Gamma_{2}, \Lambda \Rightarrow \Delta_{1} H_{1}\left|G_{2}^{* *}\right| \Pi_{1}, \Pi_{2} \Rightarrow \Delta}{\frac{H_{1}\left|G_{2}^{* *}\right| \Gamma_{1}, \Pi_{1} \Rightarrow \Delta_{1} \mid \Gamma_{2}, \Pi_{2}, \Lambda \Rightarrow \Delta}{(\mathrm{com})} H_{1} \mid \Pi, \Lambda^{k-1} \Rightarrow t}(\mathrm{com})
$$

The case of (com) with one $p p$-component on a premise and two in the conclusion is similar. Assume now that the last rule $(r)$ applied in a derivation of $H$ is a nonlinear rule or $(m g l)$ and that its conclusion is a $p p$-component, i.e. we have

$$
\frac{G_{2}\left|P_{1} \quad \ldots \quad G_{2}\right| P_{m}}{G_{2} \mid \Pi_{1}, \Sigma_{1}, \ldots, \Sigma_{n}, p^{s} \Rightarrow p}(r)
$$

By (a) in Lemma 1 , each $P_{j}$ can have one of the following forms: either $\Pi_{1}, \Xi_{j}, p^{m_{j}} \Rightarrow p$ (say the $P_{j}$ 's with $j \in\{1, \ldots, l\}$ , with $l \leq m$ ), or $\Pi_{1}, \Xi_{j} \Rightarrow p$ (say the $P_{j}$ 's with $j \in$ $\{l+1, \ldots, r\}$, with $r \leq m)$, or $\Xi_{j} \Rightarrow$. The i.h. gives us derivations of $H_{1}\left|G_{2}^{* *}\right| P_{1}^{* *}, \ldots, H_{1}\left|G_{2}^{* *}\right| P_{m}^{* *}$. In particular, this means that, for $G_{2} \mid P_{j}$, with $j \in\{1, \ldots, l\}$, we have derivations of $H_{1}\left|G_{2}^{* *}\right| \Pi_{1}, \Xi_{j}, \Lambda^{m_{j}-1}, \Pi, \Lambda^{k-1} \Rightarrow t$. The i.h. applied to the premises of $(r)$ of the form $G_{2} \mid \Pi_{1}, \Xi_{j} \Rightarrow p$ leads to derivations of $H_{1}\left|G_{2}^{* *}\right| \Pi_{1}, \Xi_{j} \Rightarrow \Delta$. From the latter, recalling that $H_{1} \mid \Pi, \Lambda^{k-1} \Rightarrow t$ is derivable, we get

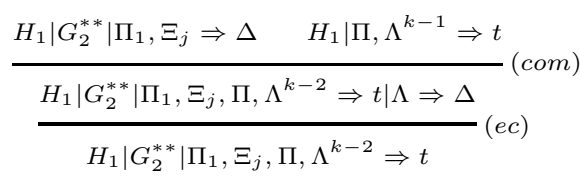

Summing up for any premise of the form $G_{2} \mid \Pi_{1}, \Xi_{j}, p^{m_{j}} \Rightarrow p$ we have a derivation of $H_{1}\left|G_{2}^{* *}\right| \Pi_{1}, \Xi_{j}, \Lambda^{m_{j}}, \Pi, \Lambda^{k-2} \Rightarrow t$, for $G_{2} \mid \Pi_{1}, \Xi_{j} \Rightarrow p$, a derivation of $H_{1}\left|G_{2}^{* *}\right| \Pi_{1}, \Xi_{j}, \Pi, \Lambda^{k-2} \Rightarrow t$ and for any premise $G_{2} \mid S_{j}$ with $R\left(S_{j}\right)=\emptyset$, a derivation of $H_{1}\left|G_{2}^{* *}\right| P_{j}^{* *}$. We can then apply the rule $(r)$ as follows:

$$
\begin{gathered}
\left\{H_{1}\left|G_{2}^{* *}\right| \Pi_{1}, \Xi_{j}, \Pi, \Lambda^{k-2} \Rightarrow t\right\}_{j=l+1, \ldots, r} \quad\left\{H_{1}\left|G_{2}^{* *}\right| P_{j}^{* *}\right\}_{j=r+1, \ldots, m} \\
\frac{\left\{H_{1}\left|G_{2}^{* *}\right| \Pi_{1}, \Xi_{j}, \Lambda^{m_{j}}, \Pi, \Lambda^{k-2} \Rightarrow t\right\}_{j=1, \ldots, l}}{H_{1}\left|G_{2}^{* *}\right| \Pi_{1}, \Sigma_{1}, \ldots, \Sigma_{n}, \Lambda^{s}, \Pi, \Lambda^{k-2} \Rightarrow t}(r)
\end{gathered}
$$

This a correct application of $(r)$. Indeed, apart from the hypersequent context, it is the original rule application, where $\Pi_{1}$ is replaced by $\Pi_{1}, \Pi, \Lambda^{k-2}$, each $p$ on the left is replaced by $\Lambda$ and each $p$ on the right by $t$. Notice that what we derived above can also be written as $H_{1}\left|G_{2}^{* *}\right| \Pi_{1}, \Sigma_{1}, \ldots, \Sigma_{n}, \Lambda^{s-1}, \Pi, \Lambda^{k-1} \Rightarrow t$ which is the desired hypersequent.

Lemma 4: Let HL and $d$ be as in Theorem 1 and assume we have a derivation of a hypersequent $G_{1} \mid \Pi \Rightarrow \Delta$ where no $p$ appears. We can find a $(D)$-free derivation of the hypersequent $H_{1}=G|\Lambda \Rightarrow \Delta| G_{1} \mid \Pi, \Lambda \Rightarrow \Delta$

Proof: Let $H$ be $H=S_{1}|\ldots| S_{n}$, we define $H^{* *}$ as $S_{1}^{* *}|\ldots| S_{n}^{* *}$ where for each $i=1, \ldots, n$

- $S_{i}^{* *}=\left(S_{i}\left[{ }^{p \Rightarrow p} / \Pi \Rightarrow \Delta\right]\right)[\bar{p} \Rightarrow / \Rightarrow]$, if $S_{i}$ is a $p p$-component.

- $S_{i}^{* *}=S_{i}\left[^{\bar{p} \Rightarrow p} / \Lambda \Rightarrow \Delta\right]$ otherwise

The statement of the lemma is a consequence of the following:

Claim: For each hypersequent $H$ in $d$ we can find a $(D)$-free derivation of $H_{1} \mid H^{* *}$

which is proved by induction on the length of the derivation $d$. If $H=p \Rightarrow p$ then $H_{1} \mid \Pi \Rightarrow \Delta$ is derivable by applying $(\overline{e w})$ to the hypersequent $G_{1} \mid \Pi \Rightarrow \Delta$ which is derivable by assumption. For logical rules, $(e c)$ and $(e w)$, the proof is similar to that of the previous lemma. Assume now that the last applied rule in a derivation of $H$ is $(\mathrm{com})$. We discuss some problematic cases involving $p p$-components, the remaining ones being similar or trivial. First, we assume that the conclusion does not contain any active $p p$-component, while one of the premises does. We have:

$$
\frac{G_{2}\left|\Gamma_{1}, \Gamma_{2}, p^{k} \Rightarrow p \quad G_{2}\right| \Pi_{1}, \Pi_{2} \Rightarrow \Delta_{1}}{G_{2}\left|\Gamma_{1}, \Pi_{1}, p^{k} \Rightarrow \Delta_{1}\right| \Gamma_{2}, \Pi_{2} \Rightarrow p}(\mathrm{com})
$$

By i.h. we have derivations of $H_{1}\left|G_{2}^{* *}\right| \Pi_{1}, \Pi_{2} \Rightarrow \Delta_{1}$ and $H_{1}\left|G_{2}^{* *}\right| \Gamma_{1}, \Gamma_{2}, \Pi \Rightarrow \Delta$. We restructure the derivation as 
follows (the last application of $(e c)$ is justified by the fact that $\Pi, \Lambda \Rightarrow \Delta$ is in $H_{1}$ )

$$
\begin{aligned}
& \frac{H_{1}\left|G_{2}^{* *}\right| \Gamma_{1}, \Gamma_{2}, \Pi \Rightarrow \Delta}{H_{1}\left|G_{2}^{* *}\right| \Gamma_{1}, \Gamma_{2}, \Gamma_{2}, \Pi \Rightarrow \Delta}(m g l) \frac{H_{1}\left|G_{2}^{* *}\right| \Pi_{1}, \Pi_{2} \Rightarrow \Delta_{1}}{H_{1}\left|G_{2}^{* *}\right| \Pi_{1}, \Pi_{2}, \Pi_{2} \Rightarrow \Delta_{1}}(m g l) \\
& H_{1}\left|G_{2}^{* *}\right| \Gamma_{1}, \Gamma_{2}, \Pi_{1}, \Pi_{2}, \Pi \Rightarrow \Delta_{1} \mid \Gamma_{2}, \Pi_{2} \Rightarrow \Delta \quad\left(\text { split }_{d}\right) \\
& H_{1}\left|G_{2}^{* *}\right| \Gamma_{1}, \Pi_{1}, \Pi, \Lambda \Rightarrow \Delta_{1}\left|\Gamma_{2}, \Pi_{2} \Rightarrow \Delta\right| \Gamma_{2}, \Pi_{2} \Rightarrow \Delta \\
& H_{1}\left|G_{2}^{* *}\right| \Gamma_{1}, \Pi_{1}, \Pi, \Lambda^{k} \Rightarrow \Delta_{1} \mid \Gamma_{2}, \Pi_{2} \Rightarrow \Delta
\end{aligned}
$$

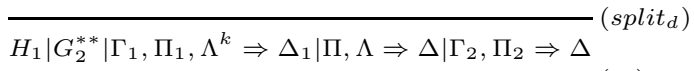

$$
\begin{aligned}
& H_{1}\left|G_{2}^{* *}\right| \Gamma_{1}, \Pi_{1}, \Lambda^{k} \Rightarrow \Delta_{1} \mid \Gamma_{2}, \Pi_{2} \Rightarrow \Delta
\end{aligned}
$$

For space reasons, in all the above applications of $(m g l)$ the single premise actually stands for two equal premises. Assume now that the conclusion of $(\mathrm{com})$ contains one active $p p$ component, while none of the premises does.

$$
\frac{G_{2}\left|\Gamma_{1}, \Gamma_{2}, p^{k} \Rightarrow \Delta_{1} \quad G_{2}\right| \Pi_{1}, \Pi_{2} \Rightarrow p}{G_{2}\left|\Gamma_{1}, \Pi_{1} \Rightarrow \Delta_{1}\right| \Gamma_{2}, \Pi_{2}, p^{k} \Rightarrow p}(\mathrm{com})
$$

Assume, w.l.o.g that $G_{2}=\emptyset$. By i.h. we have derivations of $H_{1} \mid \Gamma_{1}, \Gamma_{2}, \Lambda^{k} \Rightarrow \Delta_{1}$ and $H_{1} \mid \Pi_{1}, \Pi_{2} \Rightarrow \Delta$ from which, by applying $(\mathrm{com})$, we obtain a derivation of $H_{1} \mid \Gamma_{1}, \Pi_{1} \Rightarrow$ $\Delta_{1} \mid \Gamma_{2}, \Pi_{2}, \Lambda^{k} \Rightarrow \Delta$. Then, recalling that $H_{1} \mid \Pi \Rightarrow \Delta$ is derivable, the desired hypersequent is obtained as follows (the (ec)s are justified as $\Lambda \Rightarrow \Delta$ and $\Pi, \Lambda \Rightarrow \Delta$ are in $H_{1}$ )

$$
\begin{gathered}
\frac{H_{1}\left|\Gamma_{1}, \Pi_{1} \Rightarrow \Delta_{1}\right| \Gamma_{2}, \Pi_{2}, \Lambda^{k} \Rightarrow \Delta \quad H_{1} \mid \Pi \Rightarrow \Delta}{H_{1}\left|\Gamma_{1}, \Pi_{1} \Rightarrow \Delta_{1}\right| \Gamma_{2}, \Pi_{2}, \Pi, \Lambda^{k-1} \Rightarrow \Delta \mid \Lambda \Rightarrow \Delta}(\mathrm{com}) \\
\frac{H_{1}\left|\Gamma_{1}, \Pi_{1} \Rightarrow \Delta_{1}\right| \Gamma_{2}, \Pi_{2}, \Pi, \Lambda^{k-1} \Rightarrow \Delta}{\frac{H_{1}\left|\Gamma_{1}, \Pi_{1} \Rightarrow \Delta_{1}\right| \Gamma_{2}, \Pi_{2}, \Pi, \Lambda^{k-2} \Rightarrow \Delta \mid \Pi, \Lambda \Rightarrow \Delta}{H_{1}\left|\Gamma_{1}, \Pi_{1} \Rightarrow \Delta_{1}\right| \Gamma_{2}, \Pi_{2}, \Pi, \Lambda^{k-2} \Rightarrow \Delta}}\left(H_{1} \mid \Pi \Rightarrow \Delta\right. \\
\vdots \\
H_{1}\left|\Gamma_{1}, \Pi_{1} \Rightarrow \Delta_{1}\right| \Gamma_{2}, \Pi_{2}, \Pi \Rightarrow \Delta
\end{gathered}
$$

The case in which the last rule applied to derive $H$ is $(m g l)$ or any nonlinear rule, is handled by suitably applying the i.h., $(m g l),(\mathrm{com})$ and the rule.

\section{STANDARD COMPleteness}

Henceforth, we fix $\mathrm{L}$ to be a logic extending UL with any set $\mathcal{A}$ of nonlinear axioms and possibly mingle $\alpha^{2} \rightarrow \alpha$. For all concepts of universal algebra below we refer to [1], [8]. We call L-algebra the variety of UL-algebras satisfying the equations $\mathcal{A}^{*}$ corresponding to the axioms in $\mathcal{A}$. It follows e.g. from [1] that $\mathrm{L}$ is complete w.r.t to the class of linearly ordered L-algebras (L-chains).

Using Theorem 1, we can obtain a stronger result: the completeness of the logic $\mathrm{L}$ with respect to the class of linearly, densely ordered L-algebras (rational completeness)

Theorem 2: $\mathrm{L}$ is rational complete.

Proof: It was shown in [1], that any axiomatic extension of UL in which the following hypersequent rule

$$
\frac{G|\Lambda \Rightarrow p| \Sigma, p \Rightarrow \Delta}{G \mid \Lambda, \Sigma \Rightarrow \Delta}
$$

( $p$ eigenvariable) does not enlarge the set of provable theorems, is complete w.r.t. to the class of densely ordered Lchains. The claim follows by Theorem 1 , being the above rule derivable from the density rule considered in Section III, as follows:

$$
\begin{aligned}
& \frac{\frac{G|\Lambda \Rightarrow p| \Sigma, p \Rightarrow \Delta}{G|\Lambda, \Rightarrow p| \odot \Sigma, p \Rightarrow \Delta}(\cdot \bar{l})}{\frac{G|\Lambda, \Rightarrow p| p \Rightarrow \odot \Sigma \rightarrow \Delta}{G \mid \Lambda \Rightarrow \odot \Sigma \rightarrow \Delta}(\rightarrow r) \quad \frac{\Sigma \Rightarrow \odot \Sigma \quad \Delta \Rightarrow \Delta}{\Sigma, \odot \Sigma \rightarrow \Delta \Rightarrow \Delta}(\rightarrow l)}(\text { cut }) \\
& G \mid \Lambda, \Sigma \Rightarrow \Delta
\end{aligned}
$$

Standard completeness is then achieved through so called Dedekind Mac-Neille completion (DM-completion, for short). Indeed it is shown, e.g. in [1], that the DM-completion of a dense UL-chain is still a dense UL-chain (in other words, UL-chains are preserved by DM-completion). Moreover, the results in [14] ensure the preservation under DM-completion for the equations corresponding to acyclic $\mathcal{N}_{2}$ axioms, hence in particular for $\mathcal{A}^{*}$. The DM-completion of a dense countable $L$-chain is thus a dense $L$-chain, which in addition is orderisomorphic to $[0,1]$. This fact, together with Theorem 2 and the results in [1], leads to:

Theorem 3: $\mathrm{L}$ is standard complete.

\section{ACKNOWLEDGMENT}

This work was supported by FWF START Y544-N23 and GAP202/10/1826. We thank R. Horčík for fruitful suggestions.

\section{REFERENCES}

[1] G. Metcalfe and F. Montagna, "Substructural fuzzy logics," J. Symb. Log., vol. 72, no. 3, pp. 834-864, 2007.

[2] P. Hájek, Metamathematics of fuzzy logic. Kluwer, 1998.

[3] R. Yager and A. Rybalov, "Uninorm aggregation operators," Fuzzy Sets and Systems, vol. 80, pp. 111-120, 1996.

[4] F. Esteva and L. Godo, "Monoidal t-norm based logic: towards a logic for left-continuous t-norms," Fuzzy Sets and Systems, vol. 124, no. 3, pp. 271-288, 2001.

[5] S. Wang, "Uninorm logic with the n-potency axiom," Fuzzy Sets and Systems, vol. 205, pp. 116-126, 2012.

[6] P. Baldi, "A note on standard completeness for some extensions of uninorm logic," Soft Comput., vol. 18, no. 8, pp. 1463-1470, 2014.

[7] A. Ciabattoni, N. Galatos, and K. Terui, "From axioms to analytic rules in nonclassical logics," in LICS, IEEE, 2008, pp. 229-240.

[8] N. Galatos, P. Jipsen, T. Kowalski, and H. Ono, Residuated lattices: an algebraic glimpse at substructural logics., ser. Studies in Logics and the Foundations of Mathematics. Elesevier, 2007.

[9] G. Metcalfe, N. Olivetti, and D. Gabbay, Proof theory for fuzzy logics, ser. Springer Series in Applied Logic. Springer, 2008, vol. 36.

[10] A. Avron, "Hypersequents, logical consequence and intermediate logics for concurrency," Ann. Math. Artif. Intell., vol. 4, pp. 225-248, 1991.

[11] A. Ciabattoni and G. Metcalfe, "Density elimination," Theor. Comput. Sci., vol. 403, no. 2-3, pp. 328-346, 2008.

[12] G. Takeuti and S. Titani, "Intuitionistic fuzzy logic and intuitionistic fuzzy set theory,” J. Symb. Log., vol. 49, no. 3, pp. 851-866, 1984.

[13] P. Baldi, A. Ciabattoni, and L. Spendier, "Standard completeness for extensions of MTL: An automated approach," in WoLLIC, vol. LNCS, 2012, pp. 154-167.

[14] A. Ciabattoni, N. Galatos, and K. Terui, "Algebraic proof theory for substructural logics: Cut-elimination and completions," Ann. Pure Appl. Logic, vol. 163, no. 3, pp. 266-290, 2012. 